\title{
A SEARCH FOR LARGE VOIDS FROM COMBINED SAMPLES OF GALAXY CLUSTERS AND GALAXIES
}

\author{
K.Y. STAVREV \\ Institute of Astronomy, Bulgarian Academy of Sciences
}

\section{Introduction}

This paper is part of a more extensive study of the large voids in spatial volumes both wide and deep. Three samples of optical tracers with spectroscopically measured redshifts in a volume with $\mathrm{b} \geq 30^{\circ}$ and $\mathrm{z} \leq 0.14$ have been extracted from the catalogue of Lebedev and Lebedeva (1986) and the NASA/IPAC Extragalactic Database (NED):

- (1) a homogeneous sample of 277 rich Abell clusters

- (2) an inhomogeneous sample of 969 objects: rich and poor Abell clusters, Zwicky clusters, other clusters, and groups

- (3) a sample of 18623 NED galaxies.

\section{Void Catalog}

We have found from the normalized to $1 \mathrm{Mpc}^{3}$ distributions of the number of objects by redshift that sample 1 is fairly complete to $\mathrm{z}=0.8-0.9$, while the completeness of the subsamples of Zwicky clusters, other clusters, and groups falls sharply at about twice smaller redshifts. All samples are processed following Stavrev (1990). With the void selection criterion: void diameter $\mathrm{D} \geq 80 \mathrm{Mpc}\left(\mathrm{H}_{0}=100 \mathrm{Mpc} \mathrm{km}{ }^{-1} \mathrm{~s}^{-1}\right)$ and exclusion of "open" voids, the procedure generates a catalog of 18 and 22 large voids, respectively for samples 1 and 2 .

We have added sample 3 to sample 2 and repeated the void-search on this combined sample. The generated catalog contains 20 large galaxy voids (Table 1). Figure 1 shows the dependence of the void diameters on their distance. The increase of the void sizes in the interval above $300 \mathrm{Mpc}$ is obviously due to sample incompleteness. The dimensions of the voids 
TABLE 1. Large voids completely devoid of galaxies

\begin{tabular}{rrrrrrrrrll}
\hline $\begin{array}{r}\text { Void } \\
\text { No. }\end{array}$ & $\begin{array}{r}\alpha \\
(\mathrm{h})\end{array}$ & $\begin{array}{r}\delta \\
\left({ }^{\circ}\right)\end{array}$ & $\begin{array}{r}\mathrm{l} \\
\left({ }^{\circ}\right)\end{array}$ & $\begin{array}{r}\mathrm{b} \\
\left({ }^{\circ}\right)\end{array}$ & $\begin{array}{r}\mathrm{R} \\
(\mathrm{Mpc})\end{array}$ & $\begin{array}{r}\mathrm{D} \\
(\mathrm{Mpc})\end{array}$ & $\begin{array}{r}\mathrm{A} \\
\left({ }^{\circ}\right)\end{array}$ & $\begin{array}{r}\text { MAX } \\
(\mathrm{Mpc})\end{array}$ & e & $\begin{array}{l}\text { Percolating } \\
\text { Void(s) No. }\end{array}$ \\
\hline 1 & 8.5 & 49 & 171 & 37 & 330 & 80 & 14 & 80 & 1.0 & 2 \\
2 & 9.1 & 64 & 151 & 39 & 321 & 100 & 18 & 142 & 0.7 & 1,5 \\
3 & 9.5 & 25 & 204 & 45 & 281 & 92 & 19 & 152 & 0.6 & 4,7 \\
4 & 9.8 & 14 & 221 & 46 & 306 & 108 & 20 & 180 & 0.6 & 3,7 \\
5 & 10.6 & 50 & 162 & 56 & 338 & 80 & 13 & 80 & 1.0 & 2 \\
6 & 10.9 & -14 & 266 & 40 & 341 & 100 & 17 & 100 & 1.0 & 9 \\
7 & 11.0 & 21 & 220 & 64 & 356 & 100 & 16 & 188 & 0.5 & $3,4,8$ \\
8 & 11.1 & 29 & 203 & 67 & 391 & 86 & 13 & 86 & 1.0 & 7 \\
9 & 11.2 & -6 & 265 & 49 & 369 & 108 & 17 & 188 & 0.6 & $6,13,14$ \\
10 & 11.3 & 37 & 180 & 69 & 279 & 88 & 18 & 88 & 1.0 & \\
11 & 12.0 & -21 & 288 & 40 & 248 & 82 & 19 & 116 & 0.7 & \\
12 & 12.2 & 36 & 162 & 79 & 346 & 88 & 14 & 88 & 1.0 & \\
13 & 12.3 & -12 & 292 & 50 & 338 & 94 & 16 & 148 & 0.6 & $9,14,15$ \\
14 & 13.2 & 12 & 323 & 74 & 354 & 96 & 15 & 202 & 0.5 & $9,13,18$ \\
15 & 13.7 & -9 & 325 & 51 & 387 & 126 & 18 & 126 & 1.0 & 13 \\
16 & 13.8 & 48 & 98 & 66 & 350 & 80 & 13 & 80 & 1.0 & 17 \\
17 & 13.9 & 39 & 79 & 72 & 336 & 80 & 14 & 80 & 1.0 & 16 \\
18 & 14.2 & 12 & 0 & 65 & 331 & 100 & 17 & 169 & 0.6 & 14 \\
19 & 15.6 & 15 & 24 & 49 & 370 & 88 & 14 & 88 & 1.0 & \\
20 & 15.6 & 60 & 94 & 47 & 383 & 86 & 13 & 131 & 0.7 & \\
\hline
\end{tabular}

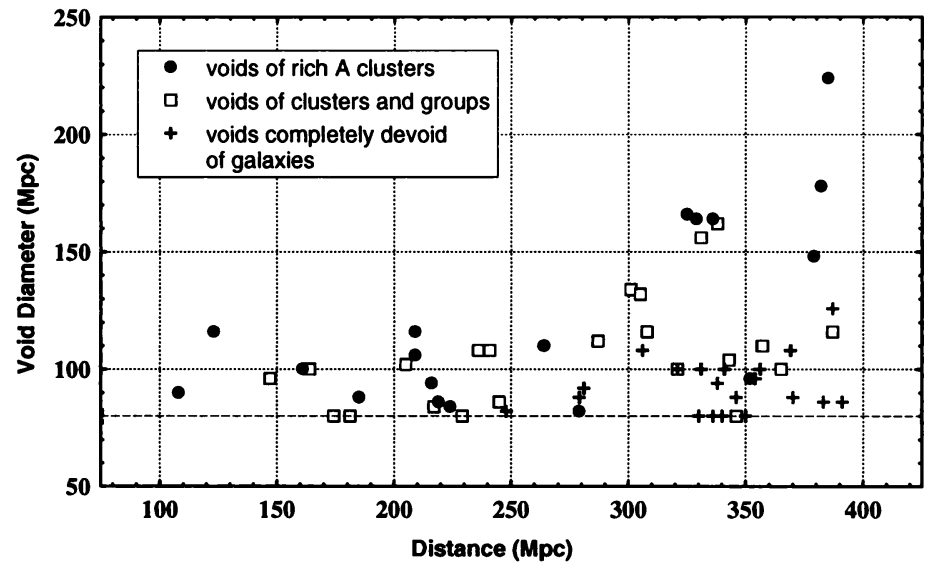

Figure 1. Void diameter versus distance 
estimated from the more complete part of the volume are about $100 \mathrm{Mpc}$, in agreement with Einasto et al. (1994). The nearer part of the volume does not contain large, completely empty voids.

\section{Acknowledgements}

This research has made use of the NASA/IPAC Extragalactic Database. Thanks are due to A. Mutafov for help in the preparation of the figure. The author thanks the SOC of Symposium 179 for support to attend it.

\section{References}

Einasto, M., Einasto, J., Tago, E., and Dalton, G. B. 1994 ESO Sci. Preprint No. 987. Lebedev, V. S., and Lebedeva, I. A. 1986 Astron. Tsirk. No. 1469, p. 4.

Stavrev, K. Y. 1990 Publ. Astron. Dep. Eötvös Univ., No. 10, p. 115. 